\title{
Exosomes as Liquid Biopsy: A Review
}

\author{
I G K N Arijana ${ }^{1}$, I G N Sri Wiryawan ${ }^{2}$, N M Linawati ${ }^{3}$ I G N Mayun ${ }^{4}$, I W Sugiritama ${ }^{5}$, \\ I G A Dewi Ratnayanti ${ }^{6}$

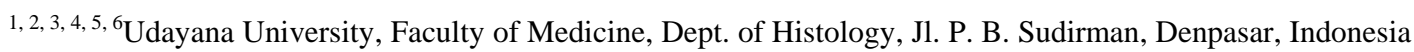

\begin{abstract}
Exosomes are nano-sized vesicles that contain protein, DNAs, mRNAs, miRNAs and ncRNAs. Their diameter is ranging from 40-100 nm and can be found in various biological fluids such as plasma, saliva, urine etc. Exosomes gain attention due to their content especially miRNAs which can regulate another cells. This regulation is important in diseases such as cancers, immune disorders, neurodegenerative diseases and infections. The review will focus in exosomal miRNA as liquid biopsy in cancers due to their advantages over traditional tissue biopsy.
\end{abstract}

Keywords: exosomes, miRNAs, liquid biopsy, tissue biopsy

\section{Introduction}

Exosomes are nano-sized vesicles that contain protein, DNAs, mRNAs, miRNAs and ncRNAs. Their diameter is ranging from $40-100 \mathrm{~nm}$ and can be found in various biological fluids such as plasma, saliva, urine etc. Exosomes gain attention due to their content especially miRNAs that can regulate another cells in diseases such as various cancers, immune disorders, neurodegenerative diseases and infections [1-3]. The review will focus in miRNAs hence called exosomal miRNA.

\section{Anatomy of Exosomes and Its Secretion}

Exosomes could be divided into its membrane and cargo. The membrane is composed of proteins and lipids similar to plasma membrane of cells. Their cargos are protein, DNAs, mRNAs, miRNAs and ncRNAs. The appearance of exosomes under electron microscopy is cup-shaped (Figure 1). Exosomes are secreted by almost cells with following orders: endosomes formation, MVBs formation and fusion with membrane cell. Regulatory systems for releasing exosomes are Rab27a, Rab27b, and interaction of syndecansyntenin-ALIX [1, 4]. An example of secreted exosomes from rat cardiomyoctes is revealed in Figure 2. Recently exosomal miRNAs gain great interest. MicroRNAs are small non-coding RNA (17-24 nucleotides) which promote gene silencing through binding to 3'UTR or open reading frame of target mRNA. The process of packaging miRNAs into exosome is a complex mechanism [1].

\section{Mechanisms of Action}

Exosomes mechanisms of action are following order: interaction of exosomal protein membrane with receptor of target cells, fusion of membrane, and internalization of exosomes. After internalization, exosomes have two options, first merger with endosomes for distribution inside target cells or neighboring cells and second is merger with endosomes then change to lysosomes for degradation.

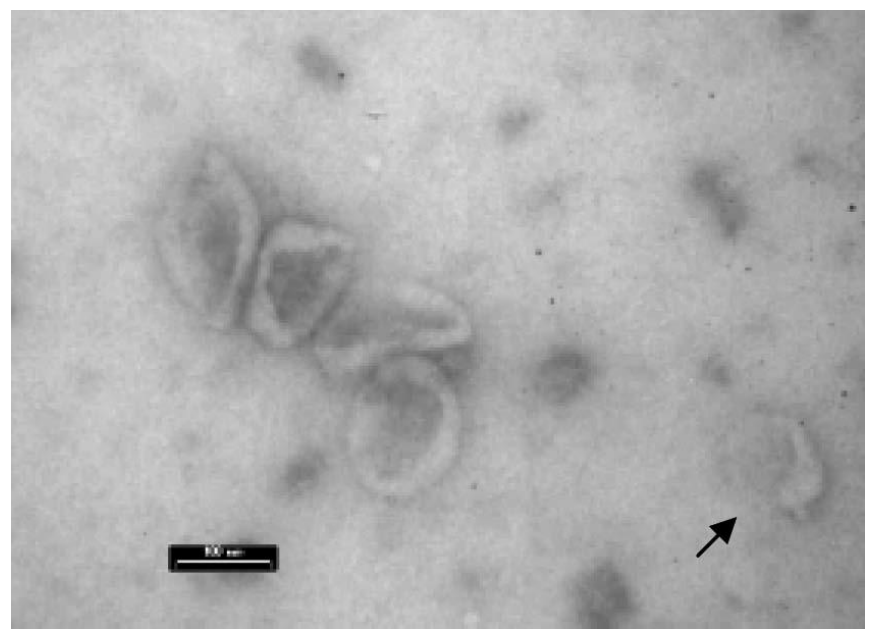

Figure 1: Cup-shaped Exosomes are Revealed by Electron Microscopy[4]

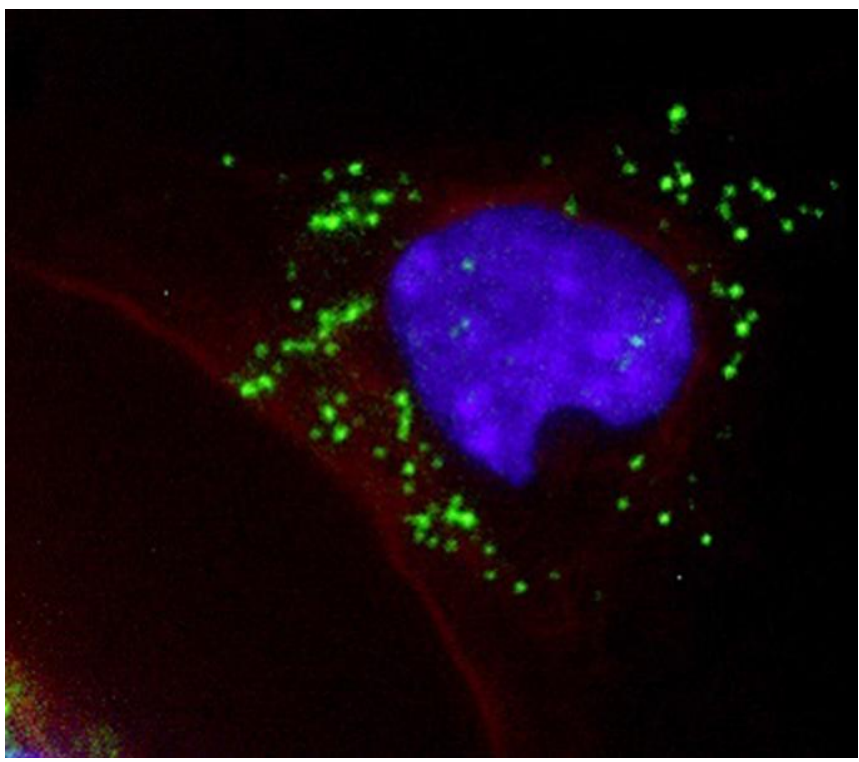

Figure 2: Exosomes (green) are secreted from rat cardiomyocytes. Nucleus is colored blue [5].

\section{Application of Exosomes as Liquid Biopsy}

Tissue biopsy is gold standard test for cancer diagnosis but the disadvantages are tissue biopsy is invasive and not possible for sampling of overall cancer process (cancer

Volume 5 Issue 6, June 2016 www.ijsr.net 


\section{International Journal of Science and Research (IJSR) \\ ISSN (Online): 2319-7064}

Index Copernicus Value (2013): 6.14 | Impact Factor (2015): 6.391

tissues are molecularly heterogeneous and evolving) [3]. Hence, non-invasive test for cancer molecular aberration another from tissue is attract a great interest, especially from blood. The term for this test is called liquid biopsy which could provides rapid and cost-effective biomarker due to allowing repeat sampling during disease progression $[3,5$, 6].

Biomarker for liquid biopsy could be derived from circulating tumor cells (CTCs), circulating free tumor nucleic acids (cfNA) and exosomes [5]. Numerous studies found that exosomes profile from healthy individuals and patients are different. The focus of liquid biopsy is exosomal miRNAs for biomarker due to their regulation function in various cancer. For instance colon cancer has exosomal miRNAs let-7a, miR-1246, miR-150, miR-21, miR-23a, miR-223 as diagnostic biomarker and prostate cancer has miR-1290, miR-375 as prognostic biomarker. Exosomal miRNA let-7f and or miR-30e-3p in Non Small Cell Lung Cancer (NSCLC) also could be used for indication of surgery. Lung adenocarcinoma has exosomal miR-17-3p, miR-21, miR-106a, miR-146, miR-155, miR-191, miR-192, miR-203, miR-205, miR-210, miR-212 and miR-214 as diagnostic biomarker [1, 4, 7-9].

\section{Conclusion}

Exosomes in peripheral blood especially exosomal miRNAs could be applied as various biomarker that non-invasive, rapid and cost-effective. This process is called liquid biopsy which is different to traditional biopsy from tissue. Liquid biopsy offers great promises for diagnostic and prognostic biomarker of cancers.

\section{References}

[1] Zhang, J., et al., Exosome and Exosomal MicroRNA: Trafficking, Sorting, and Function. Genomics, Proteomics \& Bioinformatics, 2015. 13(1): p. 17-24.

[2] Thery, C., L. Zitvogel, and S. Amigorena, Exosomes: composition, biogenesis and function. Nat Rev Immunol, 2002. 2(8): p. 569-579.

[3] He, M. and Y. Zeng, Microfluidic Exosome Analysis toward Liquid Biopsy for Cancer. J Lab Autom, 2016. 23: p. 2211068216651035.

[4] Zomer, A., et al., Exosomes: Fit to deliver small RNA. Communicative \& Integrative Biology, 2010. 3(5): p. 447-450.

[5] Sánchez-Vela, P., et al., Liquid biopsy and tumor derived exosomes in clinical practice. Revista Española de Patología, 2016. 49(2): p. 106-111.

[6] Yoshioka, Y., et al., Ultra-sensitive liquid biopsy of circulating extracellular vesicles using ExoScreen. Nat Commun, 2014. 5.

[7] Raposo, G. and W. Stoorvogel, Extracellular vesicles: Exosomes, microvesicles, and friends. The Journal of Cell Biology, 2013. 200(4): p. 373-383.

[8] Lobb, R.J., et al., Optimized exosome isolation protocol for cell culture supernatant and human plasma. 2015, 2015.

[9] Chevillet, J.R., et al., Quantitative and stoichiometric analysis of the microRNA content of exosomes.
Proceedings of the National Academy of Sciences, 2014. 111(41): p. 14888-14893.

Volume 5 Issue 6, June 2016 www.ijsr.net 